\title{
APPLICATION OF LES-STOCHASTIC TWO-WAY MODEL TO TWO-PHASE BOUNDARY LAYER FLOWS
}

\author{
Yasunori Watanabe ${ }^{1}$, Yuta Mitobe ${ }^{1}$, Yasuo Niida ${ }^{1}$ and Ayumi Saruwatari ${ }^{1}$
}

\begin{abstract}
A particle / turbulence two-way coupling model, integrated with conventional stochastic and sub-grid stress models of three-dimensional Large Eddy Simulation (LES), has been applied to the particle-laden turbulent flow in a wave boundary layer developed over seabed with the aim to understand dynamic effects of the particle size and number density to the suspension process in shearing flow over the seabed. While the particle size affects local velocity fluctuations, the particle population significantly induces secondary large-scale flows varying over a scale of the wavelength, and intensifies the turbulent energy near the bed. The particle-induced turbulence may result in additional suspension from the bed, causing a recursive suspension process via the particle turbulence interaction in the boundary layer.
\end{abstract}

Keywords: wave boundary layer, particle-fluid two-phase flow, large eddy simulation

\section{INTRODUCTION}

In shallow water, turbulence and vortices produced in a wave boundary layer disturb the sediment bottom, resulting in a complex structure of turbulent flow involving the sediments. In order to estimate the suspension load, which may predominate near-shore sediment transport, some pick-up functions and transport models have been empirically determined (e.g. Nielsen 1992), while underlying mechanisms of local suspension and sedimentation affected by turbulence have yet been understood.

There are unknown parameters to determine the suspension process; the turbulence intensity to fluidize the bed and to suspend the sediments, turbulence modification by the suspended-particles, and the particle-size dependencies on the turbulent boundary flows. Gore and Crow (1989) found a relative length of turbulence scale and particle size characterizes intensification or attenuation of the turbulent energy in particle-laden homogeneous turbulence via kinetic energy exchange between the both phases. The turbulence modification has an important role to enhance the particle dispersion and re-suspension in the particle-laden boundary layer flow under waves.

In this study, a particle-turbulence two-way model is introduced to investigate the modification of the carrier flow due to suspended particles in the wave boundary layer and to discuss possible effects of the modified turbulence on the suspension process.

\section{COMPUTATION}

In this study, the particle-laden turbulent flow was reproduced by solving the three equations system, composed of a well-known Basset-Bousinesq-Oseen equation with the stochastic Lagrangian turbulence model, the filtered momentum equation involving the filtered particle stress in LES formulation, and the sub-grid kinetic energy equation involving the particle / fluid energy interaction term (Hinze 1959).

\section{Governing Equation}

Assuming dilute particle-laden flows, the momentum equation for the liquid phase is written by

$$
\frac{\partial u_{i}}{\partial t}+\frac{\partial u_{i} u_{j}}{\partial x_{j}}=-\frac{1}{\rho_{f}} \frac{\partial p}{\partial x_{i}}+v \frac{\partial}{\partial x_{j}}\left(\frac{\partial u_{i}}{\partial x_{j}}+\frac{\partial u_{j}}{\partial x_{i}}\right)+g_{i}-\frac{1}{\rho_{f}} Q_{i}^{H},
$$

where $u_{i}$ is the fluid velocity, $\rho_{f}$ is the fluid density, $p$ is the pressure, and $g_{i}$ is the gravity vector. The particle stress $Q_{i}^{H}$ can be expressed by

$$
Q_{i}^{H}=H\left(x_{i}^{p}\right) Q_{i},
$$

where the Heaviside function is defined by $H\left(x_{i}^{p}\right)=1$ at particle location, $H\left(x_{i}^{p}\right)=0$ elsewhere (in a liquid region). Performing a filtering operation to eq. (1), we got

$$
\frac{\partial \overline{u_{i}}}{\partial t}+\frac{\partial \overline{u_{i} \bar{u}_{j}}}{\partial x_{j}}=-\frac{1}{\rho_{f}} \frac{\partial \bar{p}}{\partial x_{i}}+v \frac{\partial}{\partial x_{j}}\left(\frac{\partial \overline{u_{i}}}{\partial x_{j}}+\frac{\partial \overline{u_{j}}}{\partial x_{i}}\right)+g_{i}-\frac{\partial \tau_{i j}}{\partial x_{j}}-\frac{1}{\rho_{f}} \overline{Q_{i}},
$$

\footnotetext{
${ }^{1}$ School of Engineering, Hokkaido University, North 13 West 8, Sapporo, 060 8628, Japan
} 
where $\tau_{\mathrm{ij}}$ is the sub-grid scale (SGS) stress and the overline represents resolved variables.

The SGS kinetic energy $q_{s g s}=\frac{1}{2}\left(\overline{u_{i} u_{i}}-\overline{u_{i}} \cdot \overline{u_{i}}\right) \equiv \frac{1}{2} \overline{u_{i}^{\prime} u_{i}^{\prime}}$ is subjected to the energy transport equation:

$$
\frac{\partial q_{s g s}}{\partial t}+\frac{\partial q_{s g} \bar{u}_{j}}{\partial x_{j}}=-\tau_{i j}\left(\frac{\partial \bar{u}_{i}}{\partial x_{j}}+\frac{\partial \overline{u_{j}}}{\partial x_{i}}\right)-\varepsilon+C_{k k} \frac{\partial}{\partial x_{j}}\left(\Delta \sqrt{q_{s g s}} \frac{\partial q_{s g s}}{\partial x_{j}}\right)+v \frac{\partial^{2} q_{s g s}}{\partial x_{j} \partial x_{j}}-\frac{1}{\rho_{f}}\left(\overline{u_{i} Q_{i}^{H}}-\overline{u_{i}} \overline{Q_{i}^{H}}\right)
$$

where the coefficient $C_{k k}$ and dissipation $\varepsilon$ are given by Yoshizawa and Horiuti (1985).

It is appropriate to employ a so-called Basset-Bousinesq-Oseen (BBO) equation, in which the Basset stress is ignored, to determine spherical particle motion.

$$
\begin{gathered}
\frac{d x_{i}^{p}}{d t}=u_{i}^{p} \\
\frac{d u_{i}^{p}}{d t}=Q_{i} \\
Q_{i}=\frac{u_{i}^{s}-u_{i}^{p}}{\tau_{p}}+\frac{\rho_{f}}{\rho_{p}} \frac{D u_{i}^{s}}{D t}+\frac{C_{a}}{2} \frac{\rho_{f}}{\rho_{p}}\left(\frac{d u_{i}^{s}}{d t}-\frac{d u_{i}^{p}}{d t}\right)+\left(1-\frac{\rho_{f}}{\rho_{p}} g_{i}\right)
\end{gathered}
$$

Here, $x_{p}$ is the particle location, $u^{p}$ is the particle velocity, $\rho_{p}$ is the particle density, $C_{a}$ is the added mass coefficient and $u^{s}$ is the instantaneous fluid velocity at $x_{p}$. The relaxation time $\tau_{p}$ is defined by

$$
\tau_{p}=\frac{\rho_{p}}{\rho_{f}} \frac{4 d}{3 C_{D}\left|u_{i}{ }^{s}-u_{i}{ }^{p}\right|}
$$

where $C_{D}$ is the drag coefficient.

Since only the spatially filtered variables can be computed in LES, the unresolved velocity $u^{s}$ at the particle location, which determines the particle stress $Q_{i}$ in eq. (7), is unable to be directly computed. In this study, the following generalized Langevin equation is assumed to describe local fluid motion within a computational cell.

$$
d u_{i}^{s}=-\frac{1}{\rho_{f}} \frac{\partial\langle p\rangle}{\partial x_{i}} d t+\left(\left\langle u_{i}^{p}\right\rangle-\left\langle u_{i}^{f}\right\rangle\right) \frac{\partial\left\langle u_{i}^{f}\right\rangle}{\partial x_{j}} d t-\frac{1}{\rho_{f}} Q_{i}-\frac{u_{i}^{s}}{T_{L, i}^{*}} d t+B_{s, i} d W_{i}+g_{i} d t
$$

where $W_{i}$ is a vector-valued Wiener process and $T_{L}{ }^{*}$ are the Lagrangian time-scale prescribed with a so-called crossing trajectory effect (Csanady, 1963). $u^{\prime}$ is fluctuating velocity of $u^{s}$.

Two-way interactions between the particle and turbulent flow can be evaluated by solving the coupled equations (3), (4), (5), (6) and (9).

\section{Computing Conditions}

The computation was performed in a rectangular box-shaped domain over a frat bed under periodic small amplitude waves (see Figure 1). The velocity and pressure of the small amplitude wave theory were given at the upper boundary, and non-slip condition was imposed at the bottom boundary. The particles were initially dispersed at random locations in the domain. The wave and particle conditions given in the computations are shown in Table 1.

The computing techniques used in the computation were identical to Watanabe et al. (2005); CIP and predictor-corrector methods for the momentum and SG kinetic energy eqs. (3) and (5), and a multi-grid method for the pressure equation obtained from eq. (3) under a free-divergence condition. 


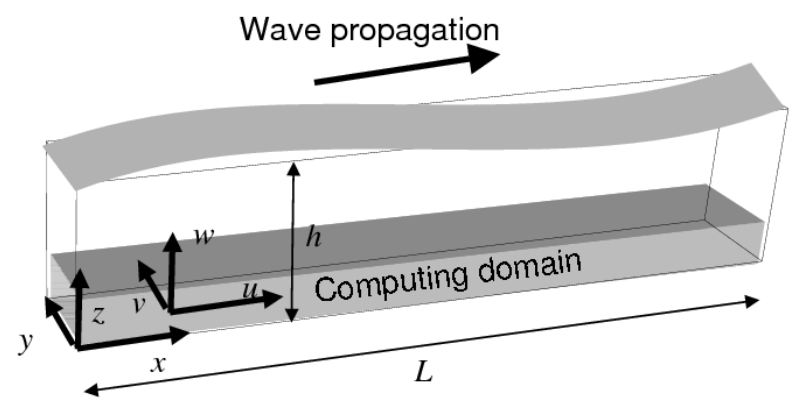

Figure 1. Computational domain and coordinates.

\begin{tabular}{|c|c|c|c|c|c|}
\hline & $\begin{array}{l}\text { Wave height } \\
H(\mathrm{~mm})\end{array}$ & $\begin{array}{l}\text { Wavelength } \\
L(\mathrm{~mm})\end{array}$ & $\begin{array}{l}\text { Specific } \\
\text { gravity }\end{array}$ & $\begin{array}{l}\text { Particle diameter } \\
d(\mathrm{~mm})\end{array}$ & $\begin{array}{l}\text { Number } \\
\text { density }(\# / l)\end{array}$ \\
\hline Run1 & $25-100$ & \multirow{6}{*}{1600} & 1.0 & \multirow{4}{*}{0.1} & \\
\hline Run2 & \multirow{5}{*}{50} & & \multirow{5}{*}{2.6} & & 180 \\
\hline Run3 & & & & & 7800 \\
\hline Run4 & & & & & 78000 \\
\hline Run5 & & & & 1.0 & \multirow{2}{*}{780} \\
\hline Run6 & & & & 0.01 & \\
\hline
\end{tabular}

\section{RESULTS}

The motion of neutral buoyant particles with specific gravity of 1.0 was computed for validating the current model. Typical ellipse-shaped trajectories under small amplitude waves were reproduced as shown in Figure 2. The major and minor axes of the computed ellipse trajectories were coincide with

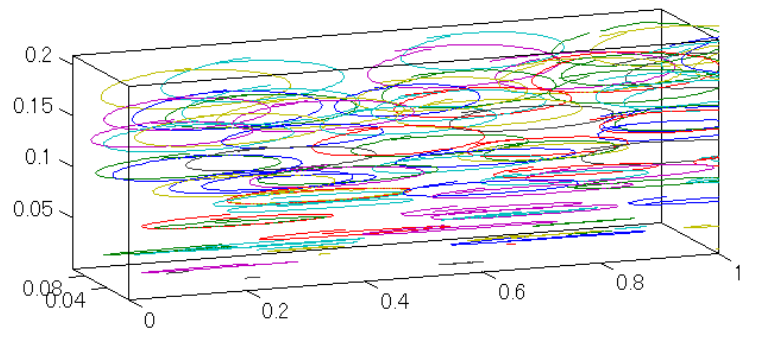

Figure 2. Particle trajectories in run 1.
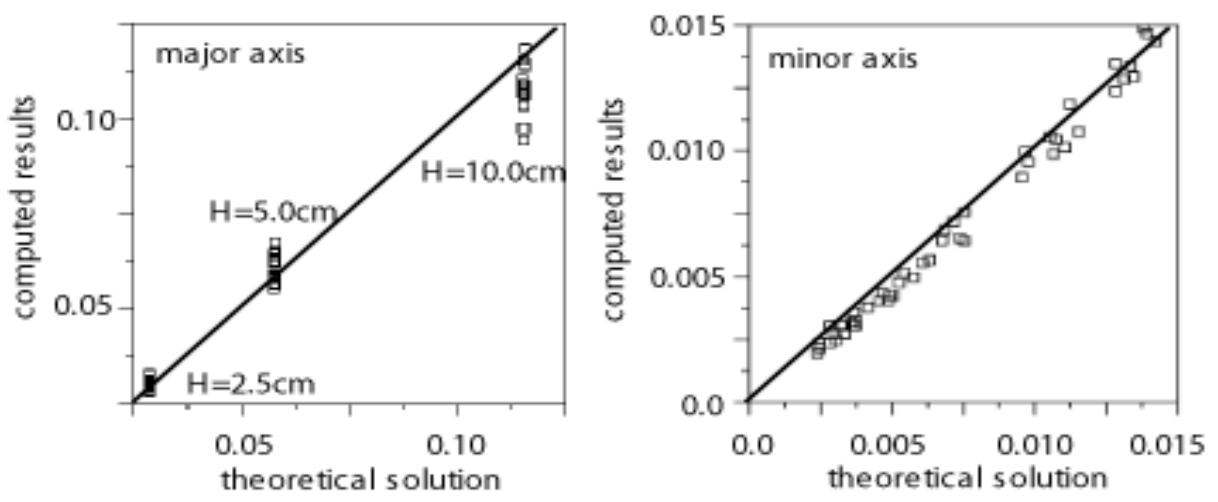

Figure 3. Major and minor axes of the computed ellipse trajectories in run 1. 
these of the small amplitude wave theory, which ensures the validity of the current computation of the particle motion (see figure 3).

Figure 4 shows the trajectories of solid particles with specific gravity of 2.6 that is identical with that of sand. Settling particle motion governs the particle flow in case of large particles $(d=1.0 \mathrm{~mm})$ since relative strength of gravity to drag is large, while smaller particles exhibit passive motion to fluid flow without settling behavior (see figure 4 bottom).

The settling particles locally disturb neighboring fluid and modify the fluid velocity. Figure 5 shows the distributions of subgrid kinetic energy in run 2. It is observed that the subgrid turbulent energy is additionally produced due to the particle drag along the particle trajectories during the settling process. It is also found that the kinetic energy is highly intensified in the vicinity of the bottom boundary where high shear is, suggesting the significant amplification of the particle-induced turbulence near the bed may affect perturbation of sediment on the bed when waves propagate over sandy bed.

Figure 6 shows the iso-surfaces of the spanwise vorticity $(\omega=\partial u / \partial z-\partial w / \partial x)$ during the settling process in run 2. It is found that the vorticity produced around the particles extends along the settling particle trajectories, and that the bottom boundary layer becomes thick when all particles have settled,
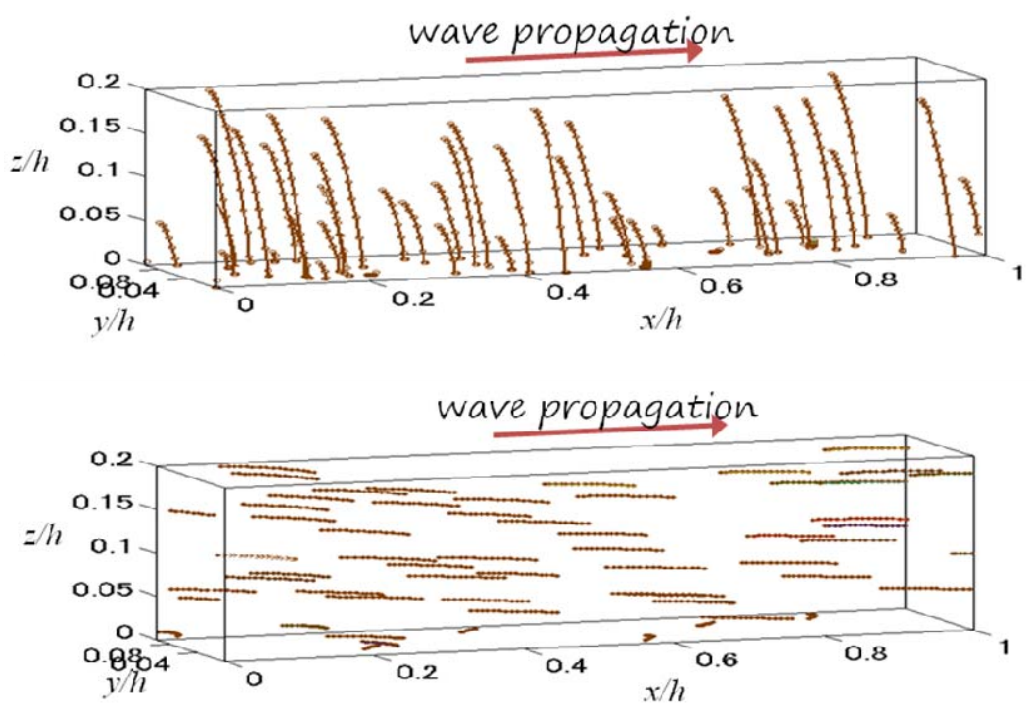

Figure 4. Particle trajectories in run2 (top) and run6 (bottom).
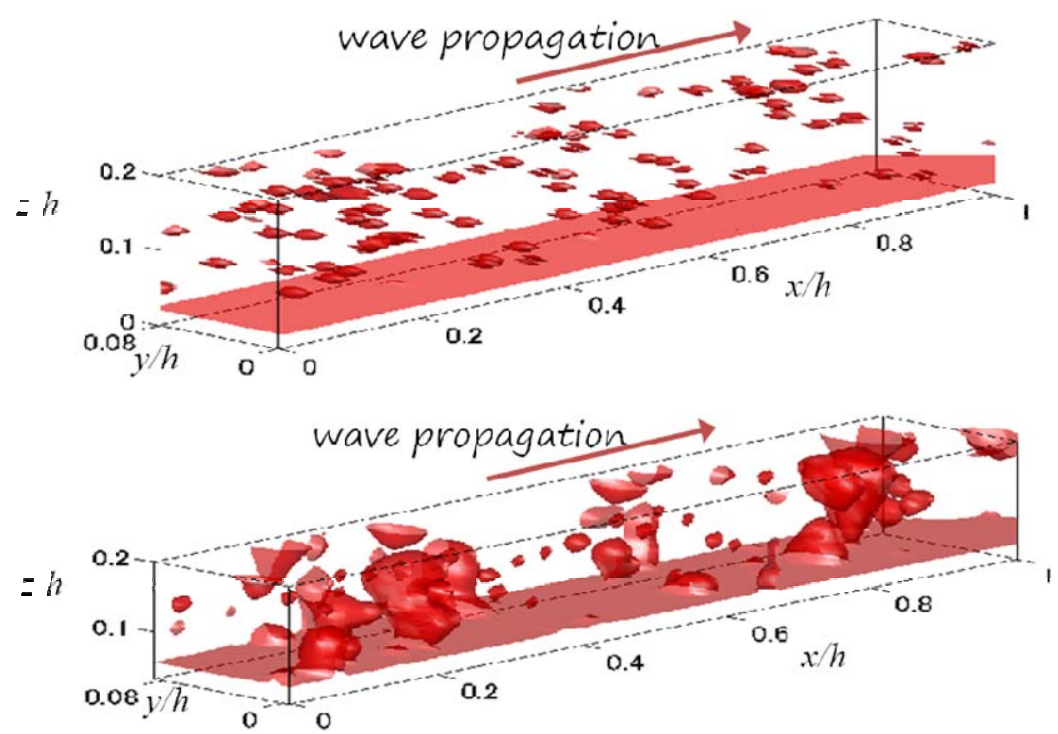

Figure 5. Iso-surfaces of subgrid kinetic energy in run 2 at the initial stage of particle release (t=T/128, top) and at the phase all particles have settled ( $t=T / 2$, bottom). 

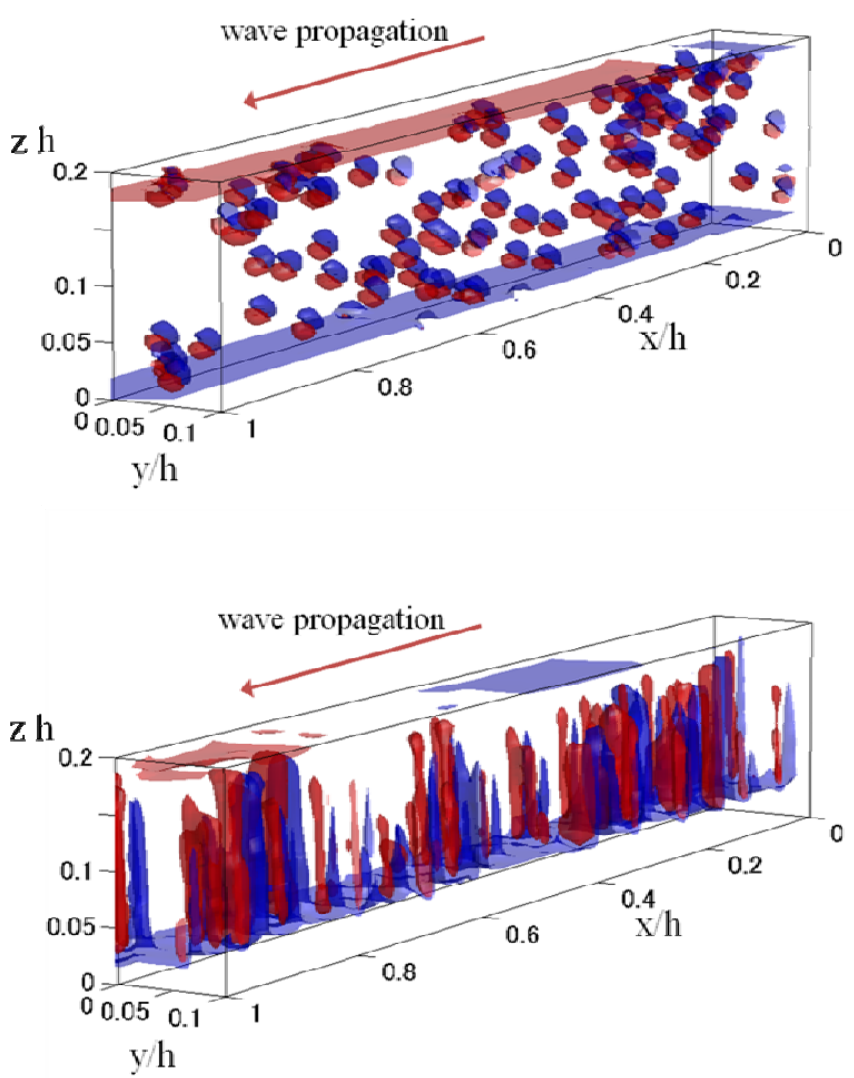

Figure 6. Iso-surfaces of spanwise vorticity in run 2 at the initial stage of particle release $(t=T / 128$, top) and at the phase all particles have settled ( $t=T / 2$, bottom).

indicating the significant contribution of the particle-driven vortex to modification of the vorticity field near the bed under progressive waves.

In order for quantitatively estimating effects of the suspended particles to turbulent flow, the modified velocities $\left(\Delta u_{i}\right)$ and turbulent energy $\left(\Delta q_{s g s}\right)$ are defined as

$$
\begin{gathered}
\Delta u_{i}=u_{i}^{+}-u_{i}^{0}, \\
\Delta q_{s g s}=q_{s g s}{ }^{+}-q_{s g s}{ }^{0},
\end{gathered}
$$

where $u_{i}^{+}$and $q_{\text {sgs }}{ }^{+}$represent the velocity and subgrid kinetic energy in the flow with suspended particles, and $u_{i}{ }^{0}$ and $q_{\text {sgs }}{ }^{0}$ are these in the simple wave boundary layer flow without particles under the same wave conditions.

Figure 7 shows the cross-sectional distributions of the modified velocity and subgrid kinetic energy over one wavelength after one wave period from the particle release. The modified vertical velocity $\left(\Delta u_{3}\right)$ appears to be negative at any wave phase, indicating the settling particles induce downward vertical fluid velocity. The modified horizontal velocity $\left(\Delta u_{1}\right)$ is negative under the wave crest and positive under the wave trough; that is, the suspended particles decelerate the horizontal carrier flow. It is also found from $\Delta q_{\text {sgs }}$ that the turbulent energy is significantly intensified near the bottom under the both of wave crest and trough. As already mentioned, this turbulence intensification in the bottom shear layer due to the particle-induced turbulence may enhance the additional disturbances of the sediment bottom and causes further suspension from the bed. This recursive interaction of the suspended particles and turbulence may be one of the important features of the suspension process in the wave boundary layer flow. 


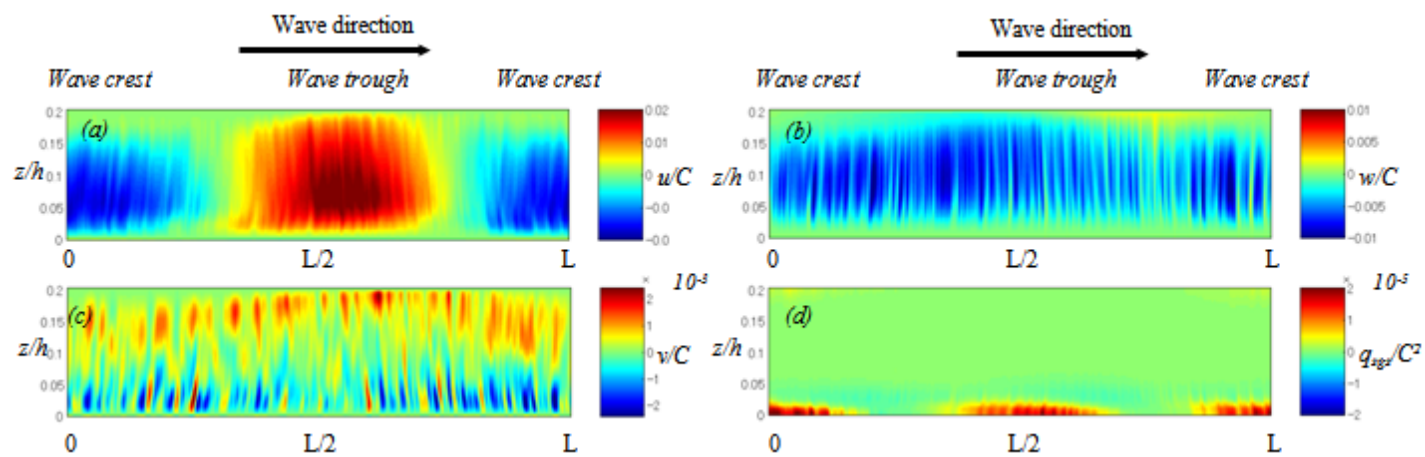

Figure 7. Cross-sectional distributions of the modified horizontal (a), vertical (b), lateral (c) velocities and subgrid kinetic energy (d) over one wavelength at $\mathrm{t}=\mathrm{T}$.

Figure 8 and 9 show the horizontal variations of the depth-integrated velocity and kinetic energy over one wavelength after one wave period from the particle release. The local fluctuations of $\Delta q_{\text {sgs }}$ and $\Delta u_{3}$ become significant as the particle diameter increases (figure 8), while the increase of particle population contributes to produce additional large-scale variations of the modified turbulence and velocity (figure 9).

The dependencies of the maximum values of the modified velocity and kinetic energy on the particle size and population are shown in Figure 10. The modified turbulent energy monotonically increase with $1 / 6$ power of the particle section area, and also with $1 / 3$ power of the number density, which indicates the additional turbulence intensification is sensitive to the particle number density rather than the particle size. Therefore, the turbulence intensity involving many small particles is
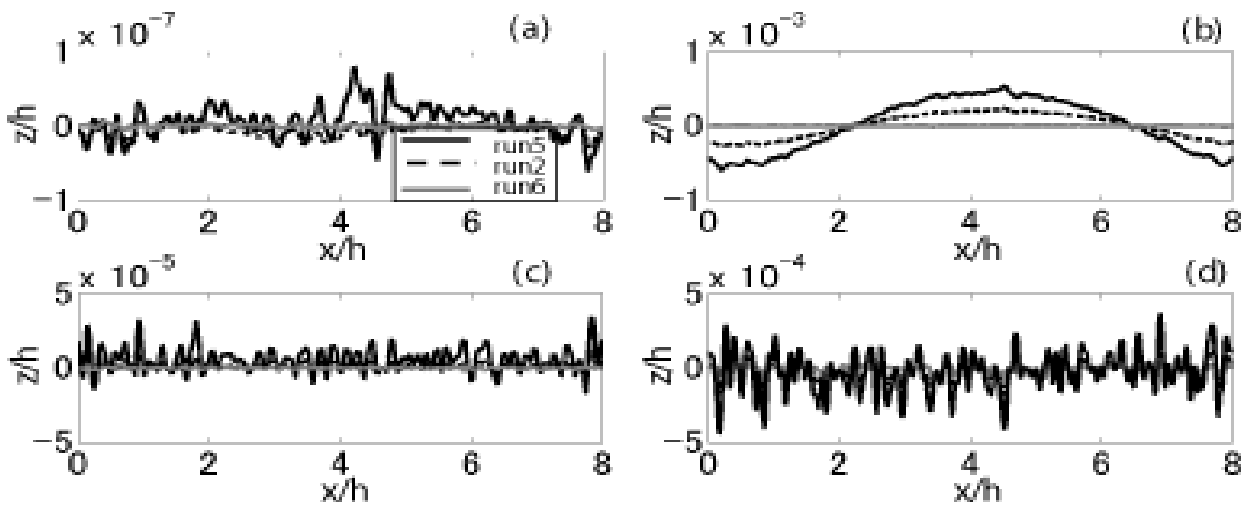

Figure 7. Horizontal variations of the depth-integrated $\Delta q_{\mathrm{sgs}}(\mathrm{a}), \Delta u_{1}(\mathrm{~b}) . \Delta u_{2}$ (c) and $\Delta u_{3}$ (d) in run2 (d=0.1mm), run5 $(d=1.0 \mathrm{~mm})$ and run $6(d=0.01 \mathrm{~mm})$ for number density of $780 r^{1}$.
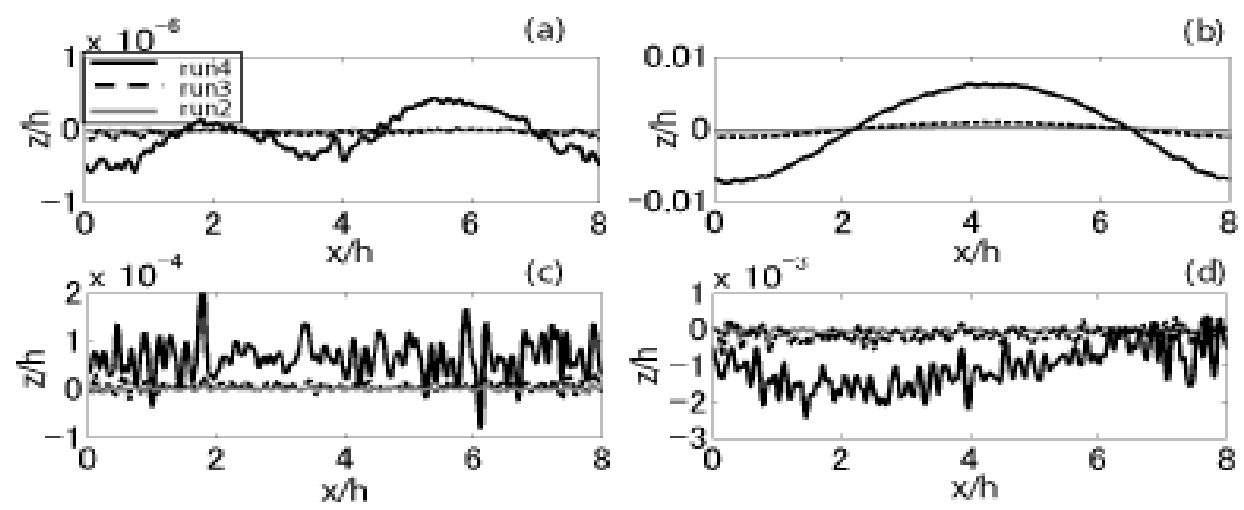

Figure 8. Horizontal variations of the depth-integrated $\Delta q_{\mathrm{sgs}}$ (a), $\Delta u_{1}$ (b). $\Delta u_{2}$ (c) and $\Delta u_{3}$ (d) in run2 (number density of $\left.780 r^{-1}\right)$, run3 $\left(7800 r^{-1}\right)$ and run $4\left(78000 r^{-1}\right)$ for particle diameter of $0.1 \mathrm{~mm}$. 

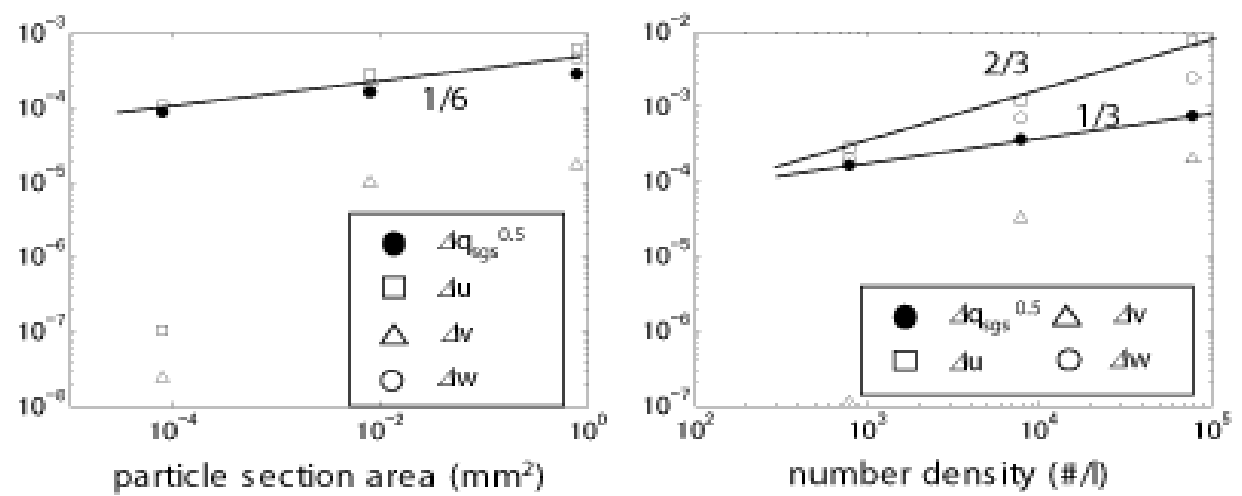

Figure 10. Maximum modified subgrid energy and velocity versus particle section area (left) and number density (right).

significantly intensified more than that for fewer and larger ones if the same volume fraction is assumed. The similar tendencies of particle size and population dependencies can be found for the modified velocity. These results suggests that the size distribution of the particles is an important factor to determine the major feature of the particle-laden wave boundary layer flow via the particleturbulence interaction in the shear flow, and that a conventional mixture model, dealing with only diffusion process of volume fraction of the sediment, may be inadequate to describe the solid-fluid two phase turbulence near the bed and the local suspension process.

\section{CONCLUSIONS}

A particle / turbulence two-way coupling model, integrated with conventional stochastic and sub-grid stress models of three-dimensional Large Eddy Simulation (LES), has been applied to the particleladen turbulent flow in a wave boundary layer developed over seabed.

This model involves consistent turbulence-particle interaction effect; the suspended particles intensify turbulence around them, while the affected turbulence modifies local structures of the carrier flow, which also changes the particle motion and drag force to produce additional turbulence again.

It has been found that, while the particle size affects local velocity fluctuations, the particle population significantly induces secondary large-scale flows varying over a scale of the wavelength, and intensifies the turbulent energy near the bed. The particle-induced turbulence may result in additional suspension from the bed, causing a recursive suspension process via the particle turbulence interaction in the boundary layer.

The intensification of the turbulent flow near the bottom was found to depend on the both of particle diameter and number density, suggesting that the size distribution of the particles is an important factor to determine the major feature of the particle-laden wave boundary layer flow via the particle-turbulence interaction in the shear flow, and that a conventional mixture model, dealing with only diffusion process of volume fraction of the sediment, may be inadequate to describe the solidfluid two phase turbulence near the bed..

\section{REFERENCES}

Csanady, G.T. 1963. Turbulent diffusion of heavy particles in the atmosphere, J. Atmos. Sci., 20, pp 201.

Gore, R. A. and C.T. Crowe. 1989. Effect of particle size on modulating turbulent intensity, Int. J. Multiphase Flow, 15, pp.279-285.

Hinze J.O. 1959. Turbulence, Macgraw-hill, pp. 790.

Nielsen P. 1992. Coastal bottom boundary layers and sediment transport, World Scientific, River Edge, N.J., pp.225.

Watanebe Y., H. Saeki, R.J. Hosking. 2005. Three-dimensional vortex structures under breaking waves, J. Fluid Mech., 291-328.

Yoshizawa, A and K. Horiuti. 1985. A statistically-derived subgrid-scale kinetic energy model for the large-eddy simulation of turbulent flows, J. Phys. Soc. Japan, 54, pp. 2834-2839. 